\title{
ERGODIC MEASURES FOR THE IRRATIONAL ROTATION ON THE CIRCLE
}

\author{
WILLIAM MORAN
}

(Received 25 March 1987)

Communicated by J. F. Price

Dedicated to Robert Edwards in recognition of

25 years' distinguished contribution to mathematics in Australia, on the occasion of his retirement

\begin{abstract}
Riesz products are employed to give a construction of quasi-invariant ergodic measures under the irrational rotation of $\mathbf{T}$. By suitable choice of the parameters such measures may be required to have Fourier-Stieltjes coefficients vanishing at infinity. We show further that these are the unique quasi-invariant measures on $\mathbf{T}$ with their associated Radon-Nikodym derivative.
\end{abstract}

1980 Mathematics subject classification (Amer. Math. Soc.): 28 D 99, 42 A 38.

\section{Introduction}

Fix an irrational number $\alpha \in(0,1)$ and consider the action $T_{\alpha}(x)=x+\alpha$ (mod 1$)$ on the circle group $T$. Lebesgue measure is well known to be the unique invariant measure for this action and it is ergodic. The question arises as to whether there exist singular quasi-invariant measures which are ergodic for the action of $T_{\alpha}$. Such measures allow the construction of non-monomial representations of the discrete Heisenberg group (see [1]):

$$
H=\left\{\left[\begin{array}{lll}
1 & x & z \\
0 & 1 & y \\
0 & 0 & 1
\end{array}\right]: x, y, z \in \mathbf{Z}\right\}
$$

(C) 1988 Australian Mathematical Society $0263-6115 / 88 \$ A 2.00+0.00$ 
Measures of this kind were first constructed by Michael Keane in [5]. His proof used the continued fraction expansion of $\alpha$ to identify the action of $T_{\alpha}$ with the odometer action on a subset of an infinite product space. For this action it is clear that appropriate infinite product measures have the desired properties. Roughly the same end can be obtained by choosing a sequence $\left(n_{k}\right)$ of positive integers such that the fractional part $\left\{n_{k} \alpha\right\}$ satisfies $0<\left\{n_{k} \alpha\right\}<2^{-k}$ and letting $\nu$ be the infinite convolution measure

$$
\nu=\underset{k=1}{\star} \frac{1}{2}\left(\delta\left(-\left\{n_{k} \alpha\right\}\right)+\delta\left(\left\{n_{k} \alpha\right\}\right)\right)
$$

where $\delta(x)$ denotes the unit point mass at $x$. The measure $\nu$ is not itself quasiinvariant but it is ergodic. This is easily seen from a result of Gavin Brown and the author [3, Proposition 1]. Replacing $\nu$ by a convex combination of its translates by all numbers of the subgroup $D_{\alpha}=\{j \alpha: j \in \mathbf{Z}\}$ of $\Pi$, we achieve a measure of the desired type.

A more general method for obtaining quasi-invariant ergodic measures which works for suitable actions on compact spaces $X$ has been given by Katznelson and Weiss in [4]. Specifically, they show that a homeomorphism of a compact metric space has a quasi-invariant ergodic measure if and only if it has a recurrent point, and in these circumstances it has uncountably inequivalent quasi-invariant ergodic measures. Their technique is akin to those of [3] and [5] in that it employs a Cantor-type construction.

In some recent work of Larry Baggett, Alan Carey, Arlan Ramsay and the author on non-monomial representations of nilpotent groups [1], there arose the problem of finding measures quasi-invariant and ergodic for the action of $T_{\alpha}$, and with Fourier-Stieltjes coefficients vanishing at infinity. Ultimately, we were able to complete the work without finding a solution to this problem. Nonetheless, it remains of some interest to construct such measures, and we do this here.

For very special irrationals it is possible to follow a method analogous to that of Keane. Let $\alpha$ be an irrational number between 0 and 1 satisfying a quadratic equation of the form $\alpha^{2}+b \alpha+c=0$ where $b, c \in \mathbf{Z}$ and $c \neq \pm 1$. Then $\alpha^{-1}$ is not a Pisot number so that by a result of Salem [7, Chapter IV, Theorem II]

$$
\nu=\underset{n=1}{\star} \frac{1}{2}\left(\delta\left(-\alpha^{n}\right)+\delta\left(\alpha^{n}\right)\right)
$$

has Fourier-Stieltjes coefficients vanishing at infinity. Moreover $\alpha^{n} \equiv k_{n} \alpha$ $(\bmod 1))$ for some $k_{n} \in \mathbf{Z}$, so that invoking the result of [3] again we can see that $\nu$ is ergodic under $T_{\alpha}$.

This method fails, however, when $c= \pm 1$, since $\alpha^{-1}$ is then a Pisot number, and so the Fourier-Stieltjes coefficients do not vanish at infinity in this case. We have no information when $\alpha$ is not a quadratic irrational as to the ergodicity of $\nu$ under $T_{\alpha}$. 
Singular measures with Fourier-Stieltjes coefficients vanishing at infinity are most easily obtained by using a Riesz product construction. Let $\left(n_{k}\right)$ be a sequence of positive integers which is lacunary in the sense that $n_{k+1} n_{k}^{-1} \geq 3$ for all $k$, and let $\left(a_{k}\right)$ be a sequence of real numbers with $0 \leq a_{k} \leq 1$. Then if

$$
P_{N}(t)=\prod_{k=1}^{N}\left(1+a_{k} \cos 2 \pi n_{k} t\right)
$$

we have $\int_{0}^{1} P_{n}(t) d t=1$ and $P_{n}(t) \geq 0$ for all $t$. The measure $\mu^{(N)}$ given by $d \mu^{(N)}(t)=P_{N}(t) d t$ is then a probability measure. Moreover, the sequence $\left(\mu^{(N)}\right)$ converges in the weak ${ }^{*}$ topology to a probability measure $\mu$. This is called a Riesz product measure (see $[8$, Chapter $\mathrm{V}, \S 7]$ ). We write

$$
d \mu(t)=\prod_{k=1}^{\infty}\left(1+a_{k} \cos 2 \pi n_{k} t\right) \cdot d t,
$$

though, in general, $\mu$ will be singular. It is a theorem of Zygmund that $\mu$ is absolutely continuous if and only if $\sum_{k=1}^{\infty} a_{k}^{2}<\infty$ (loc cit.). Its Fourier Stieltjes coefficients can be written explicitly

$$
\hat{\mu}\left(\varepsilon_{1} n_{1}+\varepsilon_{2} n_{2}+\cdots+\varepsilon_{k} n_{k}\right)=a_{1}^{\left|\varepsilon_{1}\right|} \cdot a_{2}^{\left|\varepsilon_{2}\right|} \cdots a_{k}^{\left|\varepsilon_{k}\right|}
$$

provided $\varepsilon_{i} \in\{-1,0,1\}(i=1,2, \ldots, k)$ and

$$
\hat{\mu}(r)=0 \quad \text { otherwise. }
$$

It follows that $\hat{\mu}(r) \rightarrow 0$ as $|r| \rightarrow \infty$ if and only if $a_{k} \rightarrow 0$ as $k \rightarrow \infty$.

The ergodic properties of certain Riesz products have been investigated by Gavin Brown in [2]. He showed that if $n_{k}$ divides $n_{k+1}$ for all $k$ then $\mu$ is quasiinvariant and ergodic for the action of the subgroup $D$ of $\mathbf{T}$ generated by the set $\left\{n_{k}^{-1}: k=1,2,3, \ldots\right\}$. His technique appears to rely heavily on the rigid arithmetical properties enjoyed by the $n_{k}$ 's and on the existence of appropriate finite subgroups of $D$. Of course, there are no such subgroups of $D_{\alpha}=\{j \alpha: j \in$ Z . However, by an appropriate modification of Brown's argument we are able to produce Riesz products with the desired properties.

We will show that these measures $\mu$ are uniquely defined as quasi-invariant probability measures by the Radon-Nikodym derivative $\frac{d \mu \circ T}{d \mu}$. Krieger [6] has shown that Radon-Nikodym derivatives of measures with this property form a dense $G_{\delta}$ in all such Radon-Nikodym derivatives.

This work was done while the author was a Visiting Fellow at the Centre for Advanced Study in Mathematics at Punjab University in Chandigarh. I wish to thank the members of the Centre and particularly Professor H. L. Vasudeva and his family for their kind and generous hospitality. 


\section{Construction}

We fix, in advance, a sequence $\left(a_{k}\right)$ of real numbers between 0 and $\rho$ where $0<\rho<1$. The sequence $\left(n_{k}\right)$ will be defined inductively along with a sequence $\left(\lambda_{k}\right)$ of probability measures with supports in $D_{\alpha}$. Then we shall take $\mu$ to be the Riesz product

$$
d \mu(t)=\prod_{k=1}^{\infty}\left(1+a_{k} \cos 2 \pi n_{k} t\right) \cdot d t .
$$

To facilitate the argument we make the following definitions

$$
\begin{aligned}
& P_{N}(t)=\prod_{k=1}^{N}\left(1+a_{k} \cos 2 \pi n_{k} t\right) ; \\
& d \mu_{N}(t)=\prod_{R=N+1}^{\infty}\left(1+a_{k} \cos 2 \pi n_{k} t\right) \cdot d t ; \\
& \Omega_{N}=\left\{\varepsilon_{1} n_{1}+\varepsilon_{2} n_{2}+\cdots+\varepsilon_{N} n_{N}: \varepsilon_{i} \in\{-1,0,1\}\right\} ; \\
& T_{N}=\sup \left\{r: r \in \Omega_{N}\right\} ; \\
& b_{N}=\sup \left\{|j|: j \alpha \in \operatorname{supp} \lambda_{N}\right\} .
\end{aligned}
$$

It will be convenient also to write $e_{k}(t)=\exp 2 \pi i k t$. Observe that $\mu=P_{N} \cdot \mu_{N}$, and

$$
P_{N}(t)=\sum_{r \in \Omega_{N}} P_{N}^{\wedge}(r) e_{r}(t)=\sum_{r \in \Omega_{N}} \hat{\mu}(r) e_{r}(t) .
$$

Let $\left(\varepsilon_{N}\right)$ be a sequence of real numbers with $0<\varepsilon_{N}<1$ and $\varepsilon_{N} \rightarrow 0$. We shall require $\left(n_{k}\right)$ and $\left(\lambda_{k}\right)$ to have the following properties,

$$
\left|\lambda_{N}^{\wedge}(m)\right|<\frac{1}{2} 6^{-N} \varepsilon_{N} \quad\left(0<|m| \leq 2 T_{N}\right) ;
$$

(b) for $|j| \leq b_{N}, \delta(j \alpha) * \mu_{n}$ is equivalent to $\mu_{N}$ and

$$
\left\|\delta(j \alpha) * \mu_{N}-\mu_{N}\right\|<\frac{1}{2} \varepsilon_{N} 6^{-N} .
$$

Assume, for the moment, that this has been done. We shall show that (a) and (b) together yield the ergodicity of $\mu$.

LEMMA 1. $\left\|P_{N} \cdot\left(\lambda_{N} * \mu\right)-\mu\right\|<\varepsilon_{n}$.

Proof. Let $\nu_{N}=\lambda_{N} * \mu-\mu_{N}$. Then, by (7),

$$
\begin{aligned}
\nu_{N} & =\sum_{r \in \Omega_{N}} P_{N}^{\wedge}(r) \lambda_{N} * \mu\left(e_{r} \cdot \mu_{N}\right)-\mu_{N} \\
& =\sum_{r \in \Omega_{N}} P_{N}^{\wedge}(r) \sum_{|j| \leq b_{N}} \beta_{j} \overline{e_{r}(j \alpha)} e_{r} \cdot \delta(j \alpha) * \mu_{N}-\mu_{N}
\end{aligned}
$$


where $\lambda_{N}=\sum_{|j| \leq b_{N}} \beta_{j} \delta(j \alpha)$. It follows that

$$
\begin{aligned}
\left\|\nu_{N}\right\| & \leq\left\|\sum_{r \in \Omega_{N}} P_{N}^{\wedge}(r) \sum_{|j| \leq b_{N}} \beta_{j} \overline{e_{r}(j \alpha)} e_{r} \cdot \mu_{N}-\mu_{N} l\right\| \\
& +\left\|\sum_{r \in \Omega_{N}} P_{N}^{\wedge}(r) \sum_{|j| \leq b_{N}} \beta_{j} \overline{e_{r}(j \alpha)} e_{r} \cdot\left(\delta(j \alpha) * \mu_{N}-\mu_{N}\right)\right\| \\
& \leq\left\|\sum_{r \in \Omega_{N}} P_{N}^{\wedge}(r) \lambda_{N}^{\wedge}(r) e_{r} \cdot \mu_{N}-\mu_{N}\right\|+3^{N} \sup _{|j| \leq b_{N}}\left\|\delta(j \alpha) * \mu_{N}-\mu_{N}\right\| \\
& \leq\left\|\sum_{r \in \Omega_{N}} P_{N}^{\wedge}(r) \lambda_{N}^{\wedge}(r) e_{r} \cdot \mu_{N}\right\|+2^{-N-1} \varepsilon_{N}
\end{aligned}
$$

by property (b), and by (a) this is less than $2^{-N} \varepsilon_{N}$. Now $P_{N} \cdot\left(\lambda_{N} * \mu\right)-\mu=$ $P_{N} \cdot \nu_{N}$ and $\sup _{t}\left|P_{N}(t)\right| \leq 2^{n}$ so the result follows.

We are now able to state and prove our main result, subject of course, to having found $\left(n_{k}\right)$ and $\left(\lambda_{k}\right)$ satisfying (a) and (b).

THEOREM. The measure $\mu$ of (1) is ergodic for the action of $T_{\alpha}$.

Proof. Fix a Borel set $E$ which is invariant for the action of $T_{\alpha}$, and let $r \in \mathbf{Z}$. By Lemma 1 , we have, for any $N$,

$$
\left|\int_{E} \overline{e_{r}(t)} d \mu(t)-\int_{E} \overline{e_{r}(t)} P_{N}(t) d \lambda_{N} * \mu(t)\right|<\varepsilon_{N} .
$$

Moreover, by (7),

$$
\begin{gathered}
\int_{E} \overline{e_{r}(t)} P_{N}(t) d \lambda_{N} * \mu(t)=\sum_{s \in \Omega_{N}} \hat{\mu}(s) \int_{E} e_{s-r}(t) d \lambda_{N} * \mu(t) \\
=\sum_{s \in \Omega_{N}} \hat{\mu}(s) \lambda_{N}(r-s) \int_{E} e_{s-r}(t) d \mu(t)
\end{gathered}
$$

If $N$ is sufficiently large, then $|r|<T_{N}$ and so $|r-s|<2 T_{N}$ for all $s \in \Omega_{N}$. Thus we have

$$
\int_{E} \overline{e_{r}(t)} P_{N}(t) d \lambda_{N} * \mu(t)=\hat{\mu}(r) \mu(E)+\sum_{\substack{s \in \Omega_{N} \\ s \neq r}} \hat{\mu}(s) \lambda_{N}(r-s) \int_{E} e_{s-r}(t) d \mu(t)
$$

and the final term is less in absolute value than $2^{-(N+1)} \varepsilon_{N}$. Combining this with (8) and letting $N$ tend to infinity we see that

$$
\int_{E} \overline{e_{r}(t)} d \mu(t)=\hat{\mu}(r) \mu(E)
$$

for all $r \in \mathbf{Z}$, and hence that $\mu(E)$ is 0 or 1 . 
Now we turn to the definition of $\left(n_{k}\right)$ and $\left(\lambda_{k}\right)$ to satisfy (a) and (b). Assume that $\lambda_{1}, \lambda_{2}, \ldots, \lambda_{k}$ and $n_{1}, n_{2}, \ldots, n_{k}$ have been defined to satisfy (a) and (b). Choose $n_{k+1} \geq 3 n_{k}$ such that

$$
0<\left\{n_{k+1} \alpha\right\}<\frac{(1-\rho)}{24 \pi} b_{k}^{-1} 6^{-k} \min \left(\frac{\varepsilon_{1}}{2^{k}} \frac{\varepsilon_{2}}{2^{k-1}}, \ldots, \frac{\varepsilon_{k}}{2}\right) .
$$

Define $\Omega_{k+1}$ and $P_{k+1}$ according to (2) and (4) and choose $\lambda_{k+1}$ to be a probability measure with finite support contained in $D_{\alpha}$ which is a weak * approximation to Lebesgue measure, sufficiently close for (a) to hold.

We shall use the following lemma to achieve (b).

LEMMA 2. For $|j| \leq b_{N}$, the infinite product

$$
Q_{j}^{N}(t)=\prod_{k=N+1}^{\infty}\left(\frac{1+\cos 2 \pi n_{k}(t-j \alpha)}{1+\cos 2 \pi n_{k} t}\right)
$$

converges uniformly and

$$
\left|Q_{j}^{N}(t)-1\right|<\frac{1}{2} 6^{-N} \varepsilon_{N}
$$

ProOF. We observe by (9) that if $k>N$,

$$
\begin{aligned}
\left|\left(\frac{1+a_{k} \cos 2 n_{k}(t-j \alpha)}{1+a_{k} \cos 2 n_{k} t}\right)-1\right| & \leq \frac{1}{1-\rho}\left|\cos 2 \pi n_{k}(t-j \alpha)-\cos 2 \pi n_{k} t\right| \\
& \leq \frac{2}{1-\rho}\left|\sin 2 \pi n_{k} j \alpha\right| \leq \frac{4 \pi}{1-\rho} j\left\{n_{k} \alpha\right\} \\
& \leq \frac{1}{6} 6^{-N} \varepsilon_{N} 2^{-k+N-1}
\end{aligned}
$$

Now the result follows from the general inequality

$$
\left|\prod_{k=1}^{\infty}\left(1+c_{k}\right)-1\right| \leq \exp \left(\sum_{k=1}^{\infty}\left|c_{k}\right|\right)-1, \quad \text { and } \quad \varepsilon_{N}<1 .
$$

Proposition. The measure $\mu$ is quasi-invariant for the action of $T_{\alpha}$. Furthermore, for $|j| \leq b_{N}, \delta(j \alpha) * \mu_{N}$ is equivalent to $\mu_{N}$ and

$$
\left\|\delta(j \alpha) * \mu_{N}-\mu_{N}\right\|<\frac{1}{2} 6^{-N} \varepsilon_{N}
$$


Proof. We prove first that $\delta(j \alpha) * \mu_{N}$ is equivalent to $\mu_{N}$. To see this, note that, for $f \in C(\mathbf{T})$,

$$
\begin{aligned}
& \int f(t) d \delta(j \alpha) * \mu_{N}(t)=\lim _{R \rightarrow \infty} \int_{0}^{1} f(t) \prod_{k=N+1}^{R}\left(1+a_{k} \cos 2 \pi n_{k}(t-j \alpha)\right) d t \\
& =\lim _{R \rightarrow \infty} \int_{0}^{1} f(t) \prod_{k=N+1}^{R}\left(\frac{1+a_{k} \cos 2 \pi n_{k}(t-j \alpha)}{1+a_{k} \cos 2 \pi n_{k} t}\right) \prod_{k=N+1}^{R}\left(1+\cos 2 \pi n_{k} t\right) d t \\
& =\int_{0}^{1} f(t) Q_{j}^{N}(t) d \mu(t)
\end{aligned}
$$

by Lemma 2 . It follows that

and hence that

$$
\frac{d\left(\delta(j \alpha) * \mu_{N}\right)}{d \mu_{N}}=Q_{j}^{N}(t),
$$

also by Lemma 2 .

$$
\left\|\delta(j \alpha) * \mu_{N}-\mu_{N}\right\|<\frac{1}{2} 6^{-N} \varepsilon_{N}
$$

Finally taking $j=N=1$ and noting that $\left(1+a_{k} \cos 2 \pi n_{n}(t-\alpha)\right)(1+$ $\left.a_{k} \cos 2 \pi n_{1} t\right)^{-1}$ is a continuous function bounded away from 0 , we obtain the quasi-invariance of $\mu$.

The proof that $\mu$ is quasi-invariant and ergodic is complete. If in addition we assume that $\sum_{k=1}^{\infty} a_{k}^{2}=\infty$ then $\mu$ is singular, and if $a_{k} \rightarrow 0$ as $k \rightarrow \infty$ then $\hat{\mu}$ vanishes at infinity. Thus a choice of $a_{k}=(1+k)^{-1 / 2}$ will produce a quasiinvariant ergodic singular measure with Fourier-Stieltjes coefficients vanishing at infinity. A more austere choice of $\left(a_{k}\right)$, say $a_{k}=(\log (k+1))^{-1}$, will produce a measure every convolution power of which is singular, yet $\mu$ is still ergodic and quasi-invariant for the action of $T_{\alpha}$ and has Fourier-Stieltjes coefficients vanishing at infinity.

\section{Uniqueness}

Here we show that $\mu$ is the unique quasi-invariant probability measure $\tau$ for the action of $\mathbf{T}$ such that $(d \delta(j \alpha) * \tau) / d \tau=Q_{j}\left(=Q_{j}^{0}\right)$. Observe that both sides of the equation are 1-cocycles, that is, they satisfy $\varphi_{j+k}(t)=\varphi_{j}(t-k) \varphi_{k}(t)$ for $j, k \in \mathbf{Z}, t \in \mathbf{T}$.

Suppose then that $\tau$ is such a measure and fix $r \in \mathbb{Z}$. Consider the integral

$$
I=\int e_{-r}(t) P_{M}(t) d \lambda_{M} * \nu(t)
$$

for suitably large $M$. On the one hand this is equal to

$$
\sum_{s \in \Omega_{M}} \hat{\mu}(s) \lambda_{M}^{\wedge}(r-s) \hat{\nu}(r-s)
$$


on the other, writing $\lambda_{M}=\sum_{|j| \leq b_{M}} \beta_{j} \delta(j \alpha)$, we obtain

$$
\begin{aligned}
I & =\sum_{|j| \leq b_{M}} \beta_{j} \int e_{-r}(t) P_{M}(t) d \delta(j \alpha) * \nu(t) \\
& =\sum_{|j| \leq b_{M}} \beta_{j} \int e_{-r}(t) P_{M}(t) Q_{j}(t) d \nu(t) \\
& =\sum_{|j| \leq b_{M}} \beta_{j} \int e_{-r}(t) P_{M}(t-j \alpha) Q_{j}^{M}(t) d \nu(t)
\end{aligned}
$$

by (10). It follows from Lemma 2 that

$$
\begin{aligned}
\mid I- & \sum_{|j| \leq b_{M}} \beta_{j} \int e_{-r}(t) P_{M}(t-j \alpha) d \nu(t) \mid \\
& \leq 2^{M} \sup _{t}\left|Q_{j}^{M}(t)-1\right| \leq \frac{1}{2} 3^{-M} \varepsilon_{M} .
\end{aligned}
$$

Now we observe that

$$
\begin{aligned}
J & =\sum_{|j| \leq b_{M}} \beta_{j} \int e_{-r}(t) P_{M}(t-j \alpha) d \nu(t) \\
& =\sum_{|j| \leq b_{M}} \beta_{j} \sum_{s \in \Omega_{M}} \hat{\mu}(s) \int e_{-r}(t) e_{s}(t-j \alpha) d \nu(t) \\
& =\sum_{s \in \Omega_{M}} \hat{\mu}(s) \hat{\nu}(r-s) \sum_{|j| \leq b_{M}} \beta_{j} e_{s}(-j \alpha) .
\end{aligned}
$$

Therefore,

$$
J=\sum_{s \in \Omega_{M}} \hat{\mu}(s) \hat{\nu}(r-s) \lambda_{M}(s)
$$

By (a), $\left|\lambda_{M}^{\wedge}(m)\right| \leq \frac{1}{2} 6^{-M} \varepsilon_{M}$ provided $0<|m|<2 T_{M}$, so that, by (14), if $|r|<T_{M}$,

$$
\begin{aligned}
|J-\hat{\nu}(r)| & <\sum_{\substack{s \in \Omega_{M} \\
s \neq 0}} \hat{\mu}(s) \hat{\nu}(r-s) \lambda_{M}^{\wedge}(s) \\
& \leq 2^{-(M+1)} \varepsilon_{M},
\end{aligned}
$$

whereas, by (12)

$$
|I-\hat{\mu}(r)| \leq 2^{-(M+1)} \varepsilon_{M} .
$$

Now (13), (15) and (16) combine to give $|\hat{\nu}(r)-\hat{\mu}(r)| \leq 2^{-M+1} \varepsilon_{M}$. Therefore $\hat{\mu}(r)=\hat{\nu}(r)$ for all $r \in \mathbf{Z}$ and so $\mu=\nu$. 


\section{References}

[1] L. Baggett, A. L. Carey, A. Ramsay and W. Moran, in preparation.

[2] Gavin Brown, 'Riesz products and generalised characters', Proc. London Math. Soc. (3) 27 (1973), 484-504.

[3] G. Brown and W. Moran, 'A dichotomy for infinite convolutions of discrete measures', Proc. Cambridge Philos. Soc. 73 (1973), 307-316.

[4] Y. Katznelson and B. Weiss, 'The construction of quasi-invariant measures', Istael $J$. Math. 12 (1972), 1-4.

[5] M. Keane, 'Sur les mesures quasi-ergodiques des translations irrationelles', C. R. Acad. Sci. Paris 272 (1971), 54-55.

[6] W. Krieger, 'On quasi-invariant measures in uniquely ergodic systems', Invent. Math. 14 (1971), 184-196.

[7] R. Salem, Algebraic numbers and Fourier analysis, (Heath, Boston, Mass., 1963).

[8] A. Zygmund, Trigonometric series, Vol. I, (Cambridge University Press 1959).

Department of Pure Mathematics

The University of Adelaide

Adelaide, South Australia 5000

Australia 